\title{
Zeros of Approximate Functional Approximations
}

\author{
By Robert Spira
}

1. Introduction. In this paper we discuss a calculation of zeros of

$$
g_{N}(s)=\sum_{n=1}^{N} n^{-s}+\chi(s) \sum_{n=1}^{N} n^{s-1}
$$

where

$$
1 / \chi(s)=(2 \pi)^{-s} 2(\cos (\pi s / 2)) \Gamma(s) .
$$

The $g_{N}(s)$ are of interest as approximations to the Riemann zeta function. Let $s=\sigma+i t$. In [1], it was shown that for $t$ sufficiently large, $g_{1}(s)$ and $g_{2}(s)$ have their zeros on the critical line $\sigma=\frac{1}{2}$. After encountering analytical difficulties in attempting to extend this theorem to further $N$, the calculations described below were undertaken. The results strongly suggest that for $N \geqq 3$ one can expect to find infinitely many zeros off $\sigma=\frac{1}{2}$, so that the theorem proved in [1] appears at its natural limit. For each $N$ there is a region where $g_{N}(s)$ behaves similarly to $\zeta(s)$, and also a region where it behaves similarly to $2 \zeta(s)$. This empirical information should prove very useful for work along the lines of Rouchés theorem, giving a condition for the Riemann hypothesis to be true in terms of the location of the zeros of $g_{N}(s)$.

In Section 2, we give the theory of calculating the number of zeros of an analytic function within a closed curve when the information comes from a finite number of points on the curve. The theorem requires, for application in our case, an estimate for $\left|g_{N}{ }^{\prime}(s)\right|$, and this estimate is obtained. In Section 3, the method used for calculating $\chi(s)$ is described, a difficulty being the calculation of $\Gamma(s)$ for low values of $t$. Section 4 contains a discussion of the real function $Z_{N}(t)$ analogous to the $Z(t)$ of the $\zeta$-function. In Section 5 the general organization of the calculations is described and Section 6 contains a discussion of the results. There are tables and figures of the zeros at the end.

2. The Theory of Zero Calculation. We consider first the problem of how close the spacing of points along a curve $C$ must be in order to conclude that one has counted correctly the number of zeros inside $C$ by calculating the change of argument at these points.

Let $f(z)$ be analytic inside and on a closed rectifiable Jordan curve $C$ in a region $R$. The governing theorem is that if $f(z) \neq 0$ on $C$, then the number of zeros within $C$ is given by

$$
N(f, C)=\frac{1}{2 \pi i} \int_{C} \frac{f^{\prime}(z)}{f(z)} d z=\frac{1}{2 \pi}\left[\Delta_{C} \arg f(z)\right] .
$$

By a set of sequential covering disks of $C$ we mean a finite set of closed disks $C_{1}, C_{2}$,

Received December 20, 1965. 
$\cdots, C_{n}, C_{n+1}=C_{1}$ with centers $c_{j}$ lying on $C$ whose union lies in $R$ and contains $C$ and such that the portion of $C$ lying between $c_{j}$ and $c_{j+1}$ lies wholly within $C_{j}$.

THeOREM 1. If

(i) $C_{1}, \cdots, C_{n}, C_{n+1}=C_{1}$ is a set of sequential covering disks of $C$, all of radius $h$,

(ii) $M=$ l.u.b. $\left|f^{\prime}(z)\right|, z$ in $R$,

(iii) $4 h M \leqq \min \left|f\left(c_{j}\right)\right|$,

then

$$
N(f, C)=\frac{1}{2 \pi} \sum_{j=1}^{n}\left[\arg \left(f\left(c_{j+1}\right) / f\left(c_{j}\right)\right)\right] .
$$

Proof. We need only show $f(z) \neq 0$ on $C$ and that the change of argument as we pass from $c_{j}$ to $c_{j+1}$ is $\leqq \pi / 4$. Let $z$ be on $C$ and within the disk $C_{j}$, so $z=c_{j}+z_{1}$, $\left|z_{1}\right| \leqq h$. Let $f=u+i v, z_{1}=x_{1}+i y_{1}$. Then, applying Taylor's theorem,

$$
\begin{aligned}
\left|f\left(c_{j}\right)\right|-|f(z)| & \leqq\left|f\left(c_{j}+z_{1}\right)-f\left(c_{j}\right)\right| \\
& \leqq\left|u\left(c_{j}+z_{1}\right)-u\left(c_{j}\right)\right|+\left|v\left(c_{j}+z_{1}\right)-v\left(c_{j}\right)\right| \\
& \leqq\left|x_{1} u_{x}\left(\xi_{1}\right)+y_{1} u_{y}\left(\xi_{1}\right)\right|+\left|x_{1} v_{x}\left(\xi_{2}\right)+y_{1} v_{y}\left(\xi_{2}\right)\right| \\
& \leqq\left[\left|x_{1}\right|+\left|y_{1}\right|\right] \cdot[M+M] \leqq 2 \sqrt{2} h M,
\end{aligned}
$$

since $\left|x_{1}\right|+\left|y_{1}\right| \leqq \sqrt{2}\left|z_{1}\right| \leqq \sqrt{2} h$. Hence $|f(z)| \geqq\left|f\left(c_{j}\right)\right|-2 \sqrt{2} h M$ $>0$ as $\left|f\left(c_{j}\right)\right| \geqq 4 h M$, so $f(z) \neq 0$ on $C$.

To complete the proof, note that by the above argument, all the images of the points on the curve between $c_{j}$ and $c_{j+1}$ will lie in a circle of radius $2 \sqrt{2} h M$ about $f\left(c_{j}\right)$. Since, by hypothesis, the center of this circle lies at a distance at least $4 h M$ from the origin, there can be no winding of the curve about the origin for $z$ between $c_{j}$ and $c_{j+1}$. The possible change in argument will be less if the circle with center $f\left(c_{j}\right)$ has a central distance to the origin greater than $4 h M$, and by elementary analytical geometry, at the closest position the angles of images to the central ray is at most $\pi / 4$.

If one uses McLeod's mean value theorem [2], hypothesis (iii) can be weakened to $\sqrt{2} h M \leqq\left|f\left(c_{j}\right)\right|$. According to McLeod's theorem, we can write $f\left(c_{j}+z_{1}\right)$ $-f\left(c_{j}\right)=\lambda_{1} z_{1} f^{\prime}\left(\xi_{1}\right)+\lambda_{2} z_{1} f^{\prime}\left(\xi_{2}\right), 0 \leqq \lambda_{1}, \lambda_{2} ; \lambda_{1}+\lambda_{2}=1$, and $\xi_{1}, \xi_{2}$ lying between $c_{j}$ and $c_{j}+z_{1}$. The earlier estimate then reduces to $h M$.

To apply the theorem, one cbtains an a priori estimate for $M$, and works down to an $h$ small enough to satisfy $4 h M \leqq\left|f\left(c_{j}\right)\right|$. If $f(z) \neq 0$ on $C$, such an $h$ is easily seen to exist. Setting $u_{k}+i v_{k}=f\left(c_{k}\right)$, the change of argument in (4) is easily seen to be $\arctan \left[\left(v_{k} u_{k+1}-u_{k} v_{k+1}\right) /\left(v_{k} v_{k+1}+u_{k} u_{k+1}\right)\right]$. In some cases, one may wish to locally compute the derivative and obtain only a consistent picture rather than a rigorous proof.

In our particular case, we have

$$
\left|g_{N}{ }^{\prime}(s)\right| \leqq \sum_{n=2}^{N}(\log n) n^{-\sigma}+|\chi(s)| \sum_{n=2}^{N}(\log n) n^{\sigma-1}+\left|\chi^{\prime}(s)\right| \sum_{n=1}^{N} n^{\sigma-1} .
$$

For convenience we take $t \geqq 10, \sigma>\frac{1}{2}$. With this limitation, we have, from Spira [1], Lemma 2,

$$
|\chi(s)|<1.04(|s| /(2 \pi))^{1 / 2-\sigma} .
$$


For an estimate of $\left|\chi^{\prime}(s)\right|$, we differentiate (2), obtaining

$$
\chi^{\prime}(s)=\chi(s)\left[(\pi / 2) \tan (\pi s / 2)+\log 2 \pi-\Gamma^{\prime}(s) / \Gamma(s)\right] .
$$

From Spira, [3] equation (14), we have $|\tan (\pi s / 2)| \leqq\left(1+e^{-\pi t}\right) /\left(1-e^{-\pi t}\right)$ $\leqq 1.02$ for $t \geqq 10$. From Schoenfeld [4], we have

(8) $\quad \Gamma^{\prime}(s) / \Gamma(s)=\log s-1 /(2 s)-1 /\left(12 s^{2}\right)+6 \int_{0}^{\infty} P_{3}(x) /(s+x)^{4} d x$,

and estimating as done there, we have

(9) $\left|\Gamma^{\prime}(s) / \Gamma(s)\right| \leqq|\log s|+1 /(2|s|)+1 /\left(12|s|^{2}\right)+1 /\left(10|t|^{3}\right)$.

Putting together $(6),(7)$ and $(9)$, we obtain

$$
\begin{aligned}
& \left|\chi^{\prime}(s)\right| \leqq 1.04(|s| /(2 \pi))^{1 / 2-\sigma} \\
& \quad \cdot\left[.51 \pi+\log 2 \pi+|\log s|+1 /(2|s|)+1 /\left(12|s|^{2}\right)+1 /\left(10|t|^{3}\right)\right]
\end{aligned}
$$

so that an estimate can be made for $\left|g_{N}{ }^{\prime}(s)\right|$ from (5), (6) and (10). Table I gives these estimates for $N=2,10,100, t \sim 10, t \sim 100, \frac{1}{2} \leqq \sigma \leqq 1$ and $1 \leqq \sigma \leqq 2$.

3. The $\chi$-Function. The $\chi$-function satisfies

$$
\chi(s) \chi(1-s)=1
$$

so that $\chi(s)$ can be obtained by calculating $1 / \chi(1-s)$. For the $\Gamma$-function, we use the Stirling formula (de Bruijn [5]):

$$
\Gamma(s) \sim \sqrt{ }(2 \pi) e^{-s} s^{s-1 / 2}\left[1+\sum_{k=1}^{m} a_{k} s^{-k}\right]
$$

where the $a_{k}$ are constants. Near the negative real axis, the error term for (12) becomes large, so that (12) cannot be used for both $t$ and $\sigma$ small. By repeatedly using the functional equation $\Gamma(s+1)=s \Gamma(s)$, one obtains

$$
\begin{aligned}
1 / \chi(s+8) & =(2 \pi)^{-s-8} 2(\cos (\pi s / 2)) \Gamma(s+8) \\
& =\left(\frac{s^{2}+7 s}{4 \pi^{2}}\right)\left(\frac{s^{2}+7 s+6}{4 \pi^{2}}\right)\left(\frac{s^{2}+7 s+10}{4 \pi^{2}}\right)\left(\frac{s^{2}+7 s+12}{4 \pi^{2}}\right) \frac{1}{\chi(s)},
\end{aligned}
$$

so that $\chi(s)$ can be obtained from $\chi(s+8)$ for $t$ and $\sigma$ small. We set $S=$ the

TABLE I

Estimates for $\left|g_{N}{ }^{\prime}(s)\right|$

\begin{tabular}{c|c|r|r|r}
\hline & \multicolumn{2}{|c|}{$t \sim 10$} & \multicolumn{2}{|c}{$t \sim 100$} \\
\cline { 2 - 4 } & $\frac{1}{2} \leqq \sigma \leqq 1$ & $1 \leqq \sigma \leqq 2$ & $\frac{1}{2} \leqq \sigma \leqq 1$ & $1 \leqq \sigma \leqq 2$ \\
\hline$|\chi(s)|$ & 1. & .84 & 1. & .27 \\
$\chi^{\prime}(s) \mid$ & 5.91 & 5.04 & 8.08 & 2.19 \\
$g_{2}^{\prime}(s) \mid$ & 13.01 & 16.64 & 17.35 & 7.30 \\
$g_{10}^{\prime}(s) \mid$ & 80.42 & 365.67 & 102.12 & 150.72 \\
$\mid g_{100}^{\prime}(s)$ & 1011.00 & 42903.64 & 2036.00 & 16685.40 \\
\hline
\end{tabular}


factor in brackets in (12), and separate the remaining factors of $1 / \chi(s)$ into real and imaginary parts:

$$
\begin{aligned}
1 / \chi(S)=\left[e^{y+\pi t / 2} \cos (\theta-\pi \sigma / 2)+e^{y-\pi t / 2} \cos (\theta+\pi \sigma / 2)\right. \\
\left.+i\left(e^{y+\pi t / 2} \sin (\theta-\pi \sigma / 2)+e^{y-\pi t / 2} \sin (\theta+\pi \sigma / 2)\right)\right] \cdot S
\end{aligned}
$$

where

$$
\begin{aligned}
& y=\left(\sigma-\frac{1}{2}\right) \log (|s| /(2 \pi))-\sigma-t \arg s, \\
& \theta=t \log (|s| /(2 \pi))-t+\left(\sigma-\frac{1}{2}\right) \arg s .
\end{aligned}
$$

For computation, we rearrange $e^{y+\pi t / 2}$. Since

$$
\begin{aligned}
t(\pi / 2-\arg s) & =t \arctan (\sigma / t) \\
& =\sigma\left(1-\sigma^{2} /\left(3 t^{2}\right)+\sigma^{4} /\left(5 t^{4}\right)-\sigma^{6} /\left(7 t^{6}\right)+\cdots\right)
\end{aligned}
$$

we have

$$
e^{y+\pi t / 2}=\exp \left\{\left(\sigma-\frac{1}{2}\right) \log (|s| /(2 \pi))-\sigma\left[\sigma^{2} /\left(3 t^{2}\right)-\sigma^{4} /\left(5 t^{4}\right)+\cdots\right]\right\} .
$$

For $t$ very large, it helps accuracy if the quantities $\theta \pm \pi \sigma / 2$ are computed and reduced $\bmod 2 \pi$ in double precision before computing their single precision sines and cosines.

For the $S$ factor, we write

$$
S=1+\sum_{k=1}^{m} C_{k}-i \sum_{k=1}^{m} S_{k}
$$

and use the recursion formulas

$$
\begin{aligned}
& C_{k+1}=\left(a_{k+1} / a_{k}\right)\left[C^{*} C_{k}-S^{*} S_{k}\right] /|s|, \\
& S_{k+1}=\left(a_{k+1} / a_{k}\right)\left[C^{*} S_{k}+S^{*} C_{k}\right] /|s|
\end{aligned}
$$

where $C^{*}=\cos (\arg s)$ and $S^{*}=\sin (\arg s)$. The constants $a_{k+1} / a_{k}$ may be found

\begin{tabular}{|c|c|c|c|c|}
\hline \multirow{2}{*}{$t$} & \multicolumn{2}{|c|}{$\sigma=.5$} & \multicolumn{2}{|c|}{$\sigma=1$} \\
\hline & $\operatorname{Re} \chi(s)$ & $\operatorname{Im} \chi(s)$ & $\operatorname{Re} \chi(s)$ & $\operatorname{Im} \chi(s)$ \\
\hline $\begin{array}{r}0.0 \\
1.0 \\
10.0 \\
100.0\end{array}$ & $\begin{array}{rr}1.0 & \\
-.92357 & 14911 \\
.98891 & 46004 \\
.99988 & 53642\end{array}$ & $\begin{array}{ll}0.0 & \\
-.3834262651 \\
-.1484853968 \\
-.0151412839\end{array}$ & $\begin{array}{r}-.2205391646 \\
.7852889724 \\
.2506386409\end{array}$ & \begin{tabular}{cc}
\multicolumn{2}{c}{$\infty$} \\
-.1409610581 \\
-.1078877223 \\
-.0034820602
\end{tabular} \\
\hline
\end{tabular}
in Spira [6]. Table II gives check values for $\chi(s)$ for $\sigma: .5,1 ; t: 0,1,10,100$.

4. $Z_{N}(t), \mathfrak{D}(t)$. From (1) and (11), it follows that

$$
g_{N}(s)=\chi(s) g_{N}(1-s),
$$

so, on $\sigma=\frac{1}{2}$, if $g_{N}(s) \neq 0$, we have

$$
g_{N}\left(\frac{1}{2}+i t\right) / g_{N}\left(\frac{1}{2}-i t\right)=\chi\left(\frac{1}{2}+i t\right) .
$$

TABLE II

Check values of $\chi(s)$ 


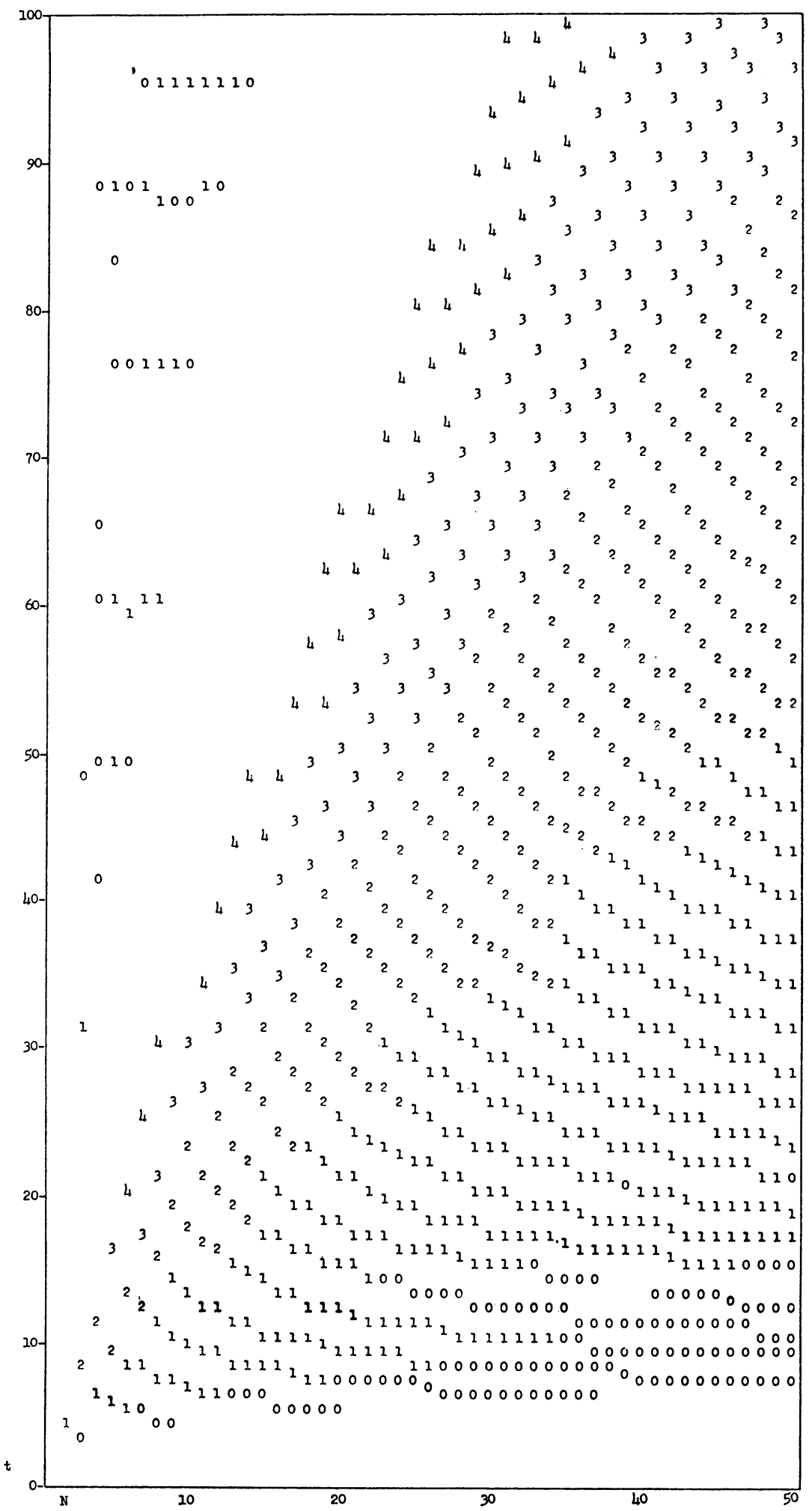

Fig. 1. Zeros of $g_{N}(s)$ 
Define

$$
\vartheta=\vartheta(t)=-\left(\arg \chi\left(\frac{1}{2}+i t\right)\right) / 2
$$

so

$$
\chi\left(\frac{1}{2}+i t\right)=e^{-2 i \vartheta}
$$

Now define

$$
Z_{N}(t)=e^{i \vartheta} g_{N}\left(\frac{1}{2}+i t\right) .
$$

If $g_{N}\left(\frac{1}{2}+i t\right) \neq 0$, then, from $(19), \arg g_{N}\left(\frac{1}{2}+i t\right) \equiv-\vartheta(\bmod 2 \pi)$, so $Z_{N}(t)$ is real for such $t$, and since it clearly vanishes exactly at the zeros of $g_{N}\left(\frac{1}{2}+i t\right)$, it is always real. It is a priori possible that $Z_{N}(t)$ be of one sign on both sides of one of its zeros. If it changes sign over an interval, then there is a zero within the interval.

As in the case of $Z(t)$ for the $\zeta$-function (Titchmarsh [7], p. 79), we find

$$
Z_{N}(t)=2 \sum_{n=1}^{N} \frac{\cos (\vartheta-t \log n)}{n^{1 / 2}}
$$

and

$$
\vartheta=\operatorname{Im} \log \Gamma\left(\frac{1}{4}+i t / 2\right)-(t \log \pi) / 2
$$

which is easily computed for $t \geq 10 \mathrm{using}$ the asymptotic series for $\log \Gamma$. For $t<10$ one can use formula (20), which will operate by the method of the previous section

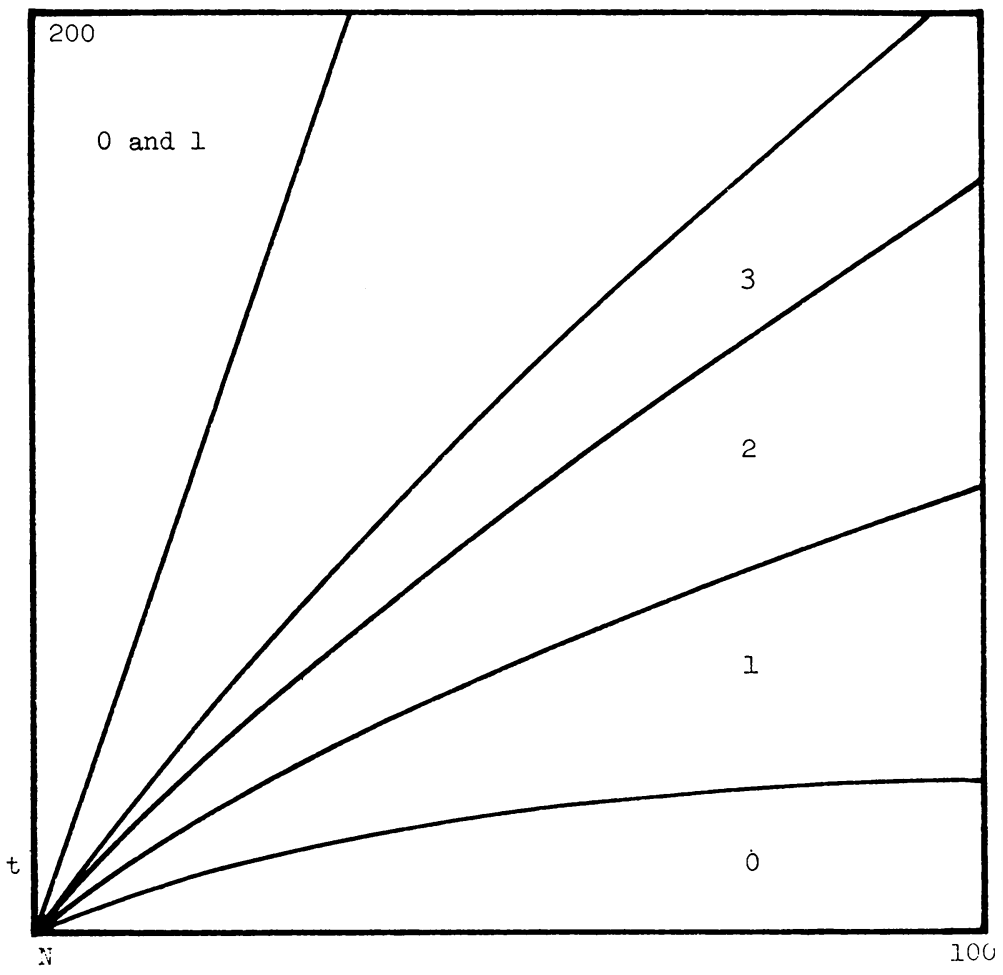

Fig. 2. Regions of zeros of $g_{N}(s)$ with given real part 
Empirically, on $\sigma=1 / 2, \chi(s)$ starts at 1 for $t=0$, and as $t$ increases, $\chi(s)$ first winds counterclockwise approximately $11 / 8$ revolutions, passing -1 near $t=.8$ and 1 near $t=3.5$ and peaking out around $t=2 \pi$, then reversing and winding ever faster clockwise as $t$ goes to infinity.

5. The Programs. Two programs were written. The first straightforwardly calculated the number of zeros within a rectangle, and printed out this number along with the minimum of the functional values calculated and the maximum change of argument between successive points on the path.

The second program was for use in the critical strip. It calculated $\Delta \arg \ddot{g}_{N}(s)$ around a rectangle centered on $\sigma=1 / 2$ by calculating $2 \Delta \arg g_{N}(s)-\Delta \arg \chi(s)$ around the right half of the rectangle. That this is correct follows from equation (18). The values of $\mathrm{g}_{N}(s)$ and $\chi(s)$ were saved, so that the values for $g_{N+1}(s)$ were easily calculated. Provision was made for expanding the rectangle and refining the step size in case unsatisfactory minima of $\left|g_{N}(s)\right|$ or unsatisfactory maxima of change of argument were obtained.

After the number of zeros within the rectangle was satisfactorily ascertained, a comparison was attempted with sign changes of $Z_{N}(t)$. The interval along the critical line could be subdivided several times to attempt to force agreement.

As the computation developed, it became clear that there were many zeros off the critical line, so that the strict requirements of Theorem 1 did not have to be applied. Thus, the limits for satisfaction were simply set small, but well above the

TABLE III

Selected zeros of $g_{N}(s)$

\begin{tabular}{r|r|r|r|r|r}
\hline$N$ & \multicolumn{1}{|c|}{ Real } & \multicolumn{1}{|c|}{ Imaginary } & $N$ & Real & Imaginary \\
\cline { 2 - 3 } \cline { 5 - 6 } 2 & 1.473596 & 4.259284 & 7 & 1.486354 & 60.035564 \\
3 & .680126 & 3.437405 & 7 & 1.214030 & 76.770971 \\
3 & 2.561720 & 8.020892 & 7 & 1.163081 & 88.014369 \\
3 & 1.403170 & 31.800344 & 7 & .855429 & 95.162091 \\
3 & .833385 & 48.882105 & 8 & 1.057658 & 60.105830 \\
4 & 1.736309 & 6.698773 & 8 & 1.224559 & 76.504505 \\
4 & 2.989619 & 11.976970 & 8 & 1.056399 & 87.836911 \\
4 & .586301 & 41.877039 & 8 & 1.287352 & 95.262405 \\
4 & .679439 & 49.068871 & 9 & 1.405330 & 76.751423 \\
4 & .855779 & 60.034343 & 9 & .739636 & 87.964248 \\
4 & .820997 & 66.058877 & 9 & 1.478086 & 95.116721 \\
4 & .549963 & 88.150047 & 10 & .826287 & 76.834502 \\
5 & 1.516003 & 49.031816 & 10 & .982439 & 87.798674 \\
5 & 1.424444 & 60.013084 & 10 & 1.161492 & 95.165571 \\
5 & .750056 & 76.458282 & 11 & 1.191464 & 88.012637 \\
5 & .893923 & 83.732473 & 11 & 1.423208 & 95.022729 \\
5 & 1.088392 & 88.219634 & 12 & .880716 & 88.106968 \\
6 & .895590 & 49.203126 & 12 & 1.498076 & 95.291697 \\
6 & 1.200929 & 59.767704 & 13 & 1.195442 & 95.358673 \\
6 & .721840 & 76.643891 & 14 & .863556 & 95.266764 \\
6 & .921079 & 88.111376 & & & \\
\hline
\end{tabular}


roundoff noise, say .05 , and the objective of the calculation was changed to obtaining a reasonably accurate picture of the true situation, with not too great an expenditure of computing time.

There was considerable cross checking between the programs. For instance, along the critical line, the program for $g_{N}(s)$ was checked against the independent programs for $Z_{N}(t)$ and $\vartheta(t)$. When roots of $\sigma=\frac{1}{2}$ were found, computing the corresponding roots with real part $1-\sigma$ gave another overall check.

6. The Zeros. Approximate locations of zeros of $g_{N}(s)$ were calculated for $N$ : 2(1) 100 in the region $\frac{1}{2}<\sigma \leqq 5,1 \leqq t \leqq 200$. Fig. 1 gives the results for $t \leqq 100, N \leqq 50$. In this figure, the integer 0 signifies a zero with real part strictly between $\frac{1}{2}$ and 1 , and the integer $k \geqq 1$ signifies a zero with real part $\geqq k$ and $<k+1$. The column of the integer signifying a zero indicates to which $N$ it belongs, and if the integer is between $t$ and $t+1$, it indicates a zero with imaginary part $\geqq t$ and $<t+1$. This $t$ is usually meant to be an integer, but in some cases of uncertainty it was chosen a half integer.

As can be seen, the majority of the zeros lie in descending chains of decreasing real part. In the portions of the chains where there is a change of the integer from $k$ to $k-1$, if there was uncertainty about whether $k$ or $k-1$ was to be chosen, the larger was taken in almost all cases.

Fig. 2 shows the boundaries of the regions where zeros having given real parts occur, the integers occurring there having the same significance as in Fig. 1. The zeros within the critical strip appear to lie outside the $t$ range $\sqrt{ }(2 \pi N) \leqq t \leqq 2 \pi N$ for each $N$. There is also a second, less obvious, $t$ range free of zeros, corresponding to where the Riemann-Siegel formula is used, $N \leqq(t / 2 \pi)^{1 / 2}<N+1$. In this second region, $g_{N}(s)$ approximates $\zeta(s)$, while in the first region, $g_{N}(s)$ is approximately $2 \zeta(s)$ since $\sum_{n=1}^{N} n^{-s}$ is approximately $\zeta(s)$ for $(2 \pi N)^{1 / 2} \leqq t \leqq 2 \pi N$, (Spira [8]), and by the functional equation, the other sum of $g_{N}(s)$ is also approximately $\zeta(s)$.

Table III gives the zeros for $N \leqq 4, t \leqq 100$, and also the zeros in the region to the left of the line $t=2 \pi N$.

The computations were done on an IBM 7040 at the University of Tennessee Computing Center, which was aided by grants NSF-G13581 and NSF-GP4046.

Department of Mathematics

University of Tennessee

Knoxville, Tennessee

1. RoBert Spira, "Approximate functional approximations and the Riemann hypothesis," Proc. Amer. Math. Soc., v. 17, 1966, pp. 314-317.

2. R. McLeOD, "Mean value theorems for vector valued functions," Proc. Edinburgh Math. Soc., v. 14, 1965, pp. 197-209.

3. Robert SpIRA, "Zero-free regions of $\zeta^{(k)}(s)$," J. London Math. Soc. v. 40, 1965, pp. $677-682$. MR 31 *5849.

4. Lowell Schoenfeld \& R. D. Dixon, "On the size of the Riemann zeta-function at places symmetric to the point $\frac{1}{2}$," Duke Math. J. 33 (1966), 291-292.

5. N. G. DE BRUIJN, Asymptotic Methods in Analysis, North-Holland, Amsterdam; Noordhoff, Groningen; Interscience, New York, 1958. MR 20, $* 6003$.

6. RoBerT SPIRA, "Calculation of the Riemann zeta function," Reviewed in Math. Comp., v. 18,1964 , pp. 519-521.

7. E. C. Titchmarsh, Theory of the Riemann Zeta Function, Oxford, at the Clarendon Press, 1951. MR 13, 741.

8. Robert SpIRA, "Zeros of sections of the zeta function. I," Math. Comp., v. 20, 1966, pp. $542-550$. 